\title{
Coherent energy migration in solids: Determination of the average coherence length in one-dimensional systems using tunable dye lasers
}

\author{
R. M. Shelby, A. H. Zewail, and C. B. Harris \\ Department of Chemistry, and Materials and Molecular Research Division of Lawrence Berkeley Laboratory, \\ University of California, Berkeley, California 94720 \\ (Received 29 September 1975)
}

\begin{abstract}
The coherent nature of energy propagation in solids at low temperatures was established from the time resolved response of the crystal to short optical pulses obtained from a dye laser (pumped by a nitrogen gas laser). The trapping and detrapping of the energy by shallow defects ( $x$ traps) was evident in the spectra and enabled us to extract the coherence length: $l \gtrsim 700 \AA=186$ molecules for the one-dimensional triplet excitons of 1,2,4,5-tetrachlorobenzene crystals at $T<4.2^{\circ} \mathrm{K}$. This length which clearly exceeds the stochastic random walk limit is related to the thermalization mechanisms in this coupled exciton-trap system, and its magnitude supports the notion that exciton-phonon coupling is responsible for the loss of coherence on very long molecular chains (trap concentration is $1 / 256000$ ).
\end{abstract}

\section{INTRODUCTION}

The problem of energy transfer in ideal crystals is simplified by the intrinsic periodic properties of the lattice which determine the explicit form for the dependence of the eigenvectors and energies on the resonance interactions between molecules in the solid. The description of the crystal states by a "quasimomentum" $k$ vector enables one to describe the dynamics of energy migration in such lattices in terms of a group velocity $V_{g}(k)$ which is determined by the energy dispersion of the band. ${ }^{1}$ In one-dimensional systems, the physics is simplified considerably. The energy dispersion is given by

$$
E(k)=E^{0}+2 \beta \cos k a,
$$

and the group velocity is

$$
V_{g}(k)=[1 / \hbar][\partial E(k) / \partial k]=(2 \beta a / \hbar) \sin k a,
$$

where $\beta$ is the nearest neighbor resonance interaction and $E^{0}$ is the energy of the molecules in the crystal site which are separated by the lattice vector $a$. Equation (2) shows that the velocity distribution is different in the different $k$ states and has a maximum value at the band center. This description, however, is only valid when the scattering between $k$ states occurs on a time long compared to the intermolecular interaction time, $\hbar / \beta$.

Dislocations, chemical impurities, and phonons play a very important role in the migration processes insofar as exciton-phonon and exciton-trap interactions destroy the coherent nature of the process and make the dynamics look more incoherent or "random" in nature. ${ }^{2-5}$ The extent to which the dynamics approach the random walk limit depends on a variety of distinct problems. In order to understand the detailed nature of exciton migration in crystals, the different roles of phonon-exciton scattering, trapping, the mechanisms for detrapping or promotion of localized excitations to the delocalized states of the exciton band, and the triplet state radiative and nonradiative depopulating channels must be determined. It is also important to examine the role temperature plays in determining the dynamics.

In the low temperature coherent limit the time between exciton-phonon scattering events is long enough so that the exciton propagation can be characterized by an average group velocity $\left\langle V_{g}\right\rangle{ }^{6-9}$ This is a temperature dependent quantity since it is a statistical average over the thermal population of the various $k$ states. Naturally this is valid only for the case where thermal equilibrium is reached on a time scale short compared to the rate of trapping. The values of $\left\langle V_{g}\right\rangle$ vary only weakly with temperature between $1^{\circ}$ and $4^{\circ} \mathrm{K}$ when the exciton bandwidth is less than $5 \mathrm{~cm}^{-1}$, whereas it may change by a factor of 2 in the same temperature range for a bandwidth of $25 \mathrm{~cm}^{-1}$. In the coherent limit the rate at which mobile excitons are localized by a trap site (trapping rate constant) is given by the constant

$$
K_{i}=\alpha\left(\left\langle V_{g}\right\rangle / d\right)
$$

where $d$ is the average distance between traps, and $i$ indicates that the excitation is going into the trap. Clearly, it is conceivable that not every exciton-trap encounter need lead to trapping of the exciton. The constant $\alpha$ represents the probability that such an encounter actually results in localization of the excitation on the trap molecule. This probability is presumably related to the exact form of the linear combination of $k$ states which makes up the exciton, the length of time the exciton interacts with the trap, and the availability of a channel or channels for dissipation of the trapexciton energy difference in the form of phonons. In view of little or no information related to these details, the absolute magnitude of $\alpha$ is difficult to estimate.

Another important limit for energy migration is the one where the frequency of exciton-phonon scattering is much greater than the nearest neighbor interaction $(\beta / \hbar)$. In this case, the exciton-phonon scattering is sufficient to localize the excitation on a single molecule, and one expects that the exciton migration will be characterized by a random walk or hopping motion with the step length equal to the intermolecular spacing 
(say a) and with the average time per step given by $t=h / 4 \beta$, where $\beta$ is the intermolecular interaction matrix element. This picture results in a trapping rate constant (inverse of the average time to reach a trap) on the order of

$$
K_{i}=a^{2} / t d^{2},
$$

which is independent of temperature.

In the intermediate regime where exciton-phonon scattering is of insufficient frequency to completely localize the excitation but does serve to diminish the coherence time of the exciton, the effect of this interaction must be included in an estimation of $K_{i}$. For example, a simple model is to assume that the exciton migration can be characterized by a random-walk motion with a step length equal to the average coherence length $\langle l\rangle$, whose upper limit is determined by $\left\langle V_{g}\right\rangle$ and the coherence time $\tau_{c}$ :

$$
\tau_{c}\left\langle V_{g}\right\rangle=\langle l\rangle \text {. }
$$

With these assumptions, one obtains

$$
K_{i}=\alpha\langle l\rangle^{2} / \tau_{c} d^{2} \leq\left(\left\langle V_{g}\right\rangle / d\right)(\langle l\rangle / d) .
$$

Alternatively, one can characterize the coherence by a time correlation function ${ }^{10}$ assuming an explicit Hamiltonian for scattering and arrive at time correlated values of $K_{i}$.

In either case, the important point to note is that the measurement of the "trapping" or localization rate constants provides a method of determining whether or not coherence is an important consideration in energy transfer processes and to determine a semiquantitative value for the coherence length.

In order to extract this information, all the kinetic rate constants for processes such as trapping, detrapping, and decay (radiative or nonradiative) and their explicit dependence on the thermal energy distribution must be determined. Conventional optical ${ }^{11}$ and magnetic resonance techniques ${ }^{12}$ can extract the decay rate constants to the ground state. The detrapping rate constant, on the other hand, was recently obtained ${ }^{13,14}$ by using the optical detection of electron spin coherence ${ }^{15}$ in localized trap states. A prerequisite for this method is that the spin system respond coherently to the applied microwave field, and consequently the spread in the frequency distribution for the resonances must be less than the microwave field strength. Moreover, the polarization of the spin should be high enough to cause a significant change in the phosphorescence intensity.

At higher temperature, however, the spin polarization will be lost, and new ways for obtaining these detrapping rate constants and exciton dynamics are desired. To this end, we have utilized short light pulses ( $5 \mathrm{nsec}$ ) obtained from a tunable dye laser to selectively excite band or localized states of one-dimensional systems while monitoring the time dependence of the light emitted from the various states. Although the approach would apply equally well to systems of higher dimensionality, the choice of the simple systems, 1 , 4-dibromonaphthalene (DBN) and 1,2,4,5-tetrachloro- benzene (TCB), serves to give an ideal case because both $\mathrm{TCB}^{16}$ and $\mathrm{DBN}^{17,18}$ have been shown to exhibit behavior characteristic of essentially one-dimensional triplet excitions at liquid helium temperatures. This allows the kinetic equations to be solved in an exact form; the group velocity and coherence time directly reflect the inchain dynamics of the exciton. The measurements of trapping rates and trap concentrations allowed a minimum coherence length to be established in the solid TCB at very low temperatures.

\section{EXPERIMENTAL}

\section{A. Sample preparation}

DBN was purchased from Eastman Organic Chemicals and zone refined for 125 passes at $1 \mathrm{in}$. $/ \mathrm{h}$. TCB obtained from Eastman was vacuum sublimed into a zone refining tube and zone refined for 1800 passes. Both crystals were then grown from the melt using Bridgman techniques.

\section{B. Experimental arrangement}

For temperatures above $4.2^{\circ} \mathrm{K}$, the crystals were mounted on a brass holder and placed in a Janis Research Corp. variable temperature Dewar where the sample was cooled by a flow of helium vapor. The temperature was monitored using a carbon resistor in contact with the crystal holder and could be regulated to $\pm 0.05^{\circ} \mathrm{K}$. The carbon resistor was calibrated against a lead-germanium thermometer. For temperatures below $4.2^{\circ} \mathrm{K}$, the sample was immersed directly in the liquid helium bath, either in the Janis Dewar or in a homemade Dewar where temperatures below $4.2{ }^{\circ} \mathrm{K}$ were reached by pumping on the helium bath with a Kinney model KTC-21 vacuum pump or a Leybold-Heraus (Type DK-200) vacuum pump.

For the time-resolved phosphorescence experiments, the first excited triplet origin or vibronic level was populated with a laser pulse of approximately 5 nsec duration from a Molectron Corp. UV-1000; DL-200 nitrogen laser-dye laser combination. PBD in $p$ dioxane and coumarin 102 in ethanol were used as the lasing dyes (obtained from Eastman Organic Chemicals) for TCB and DBN, respectively. The population of the triplet exciton or trap levels was monitored via their phosphorescence emission to the ground state origin or vibronic level using a Spex $3 / 4 \mathrm{~m}$ monochrometer and cooled $\left(-20^{\circ} \mathrm{C}\right)$ EMI $6256 \mathrm{~S}$ photomultiplier at right angles to the excitation beam. The phosphorescence signal was time averaged using a Northern NS-575 signal averager or a Biomation model 8100 transient recorder interfaced to the NS-575, depending on the desired time resolution.

For the phosphorescence temperature dependence of $\mathrm{TCB}$, the crystal was excited using a $100 \mathrm{~W}$ mercury arc lamp, filtered by a water filter and Schott $3100 \AA$ interference filter. The phosphorescence was scanned using a 3/4 $\mathrm{m}$ Spex monochrometer in second order with a resolution of approximately $0.2 \AA$ at $3131 \AA$. The temperature was controlled by varying the rate of pumping on the liquid helium and was monitored during the recording of the spectra using the calibrated car- 


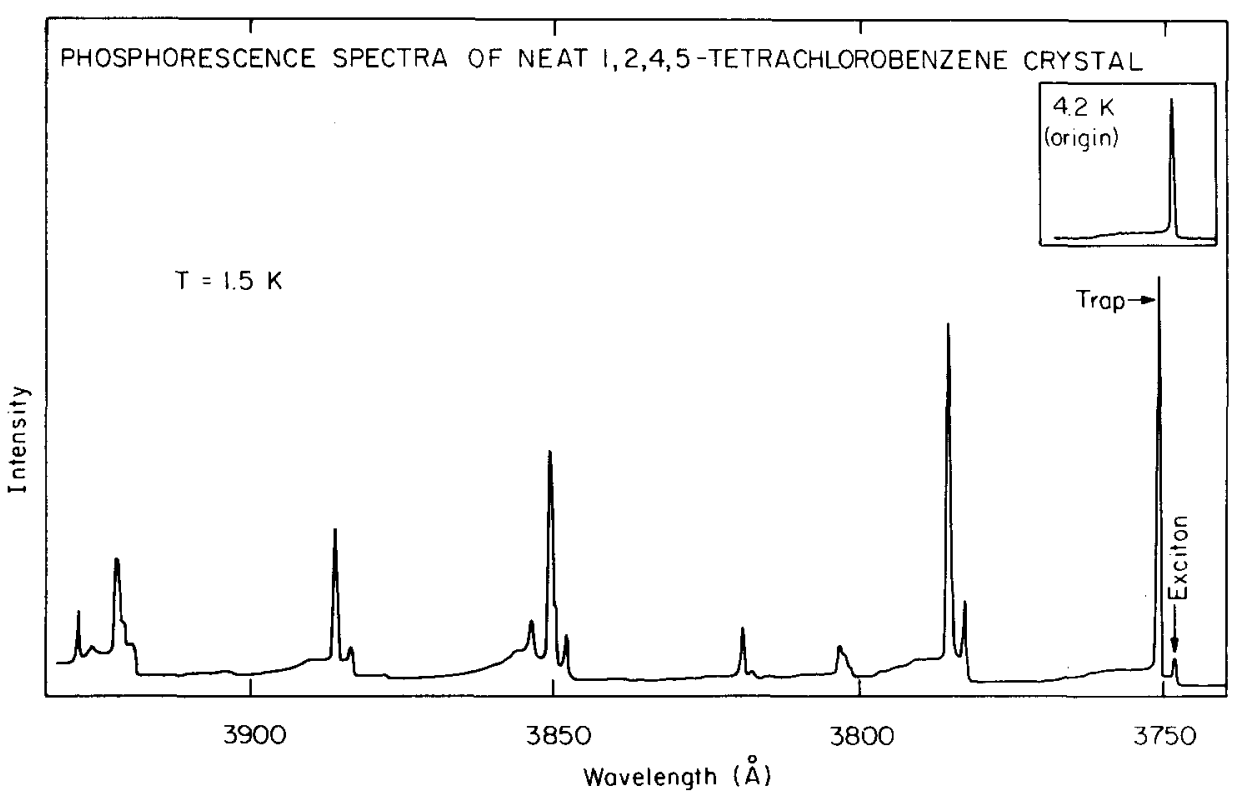

FIG. 1. Phosphorescence spectra of neat $1,2,4,5-$ tetrachlorobenzene. The exciton origin $(0,0)$ is at $3748 \AA$ and $b_{2 g}$ vibronic origin at $3781 \AA$. The $x$-trap origin $(0,0)$ is at $3751 \AA$ and the $b_{2 g}$ vibronic origin is $3784 \AA$. The inset shows the exciton emission at $4.2^{\circ} \mathrm{K}$. bon resistor. Phosphorescence spectra of 1,4-dibromonaphthalene were recorded in a similar manner.

\section{Phosphorescence of 1, 2, 4, 5-tetrachlorobenzene}

The phosphorescence spectrum of TCB at $1.5^{\circ} \mathrm{K}$ and at $4.2^{\circ} \mathrm{K}(0,0$ origin only) is shown in Fig. 1. At $4.2^{\circ} \mathrm{K}$ the phosphorescence intensity is essentially due to exciton emission. As the temperature is lowered, the $x$-trap (spectroscopic trap depth $=21 \mathrm{~cm}^{-1}$ ) population $^{19}$ increases until at $1.5^{\circ} \mathrm{K}$ the $x$-trap electronic origin emission is roughly 16 times as intense as that of the exciton origin. In addition to the exciton and $x$-trap electronic origin, several vibrational progressions are observed in the phosphorescence spectra. In particular, for many of the pulsed excitation experiments, emission from the $x$-trap or exciton $b_{2 g}$ vibronic origin was monitored (see Fig. 1).

\section{Phosphorescence of 1,4-dibromonaphthalene}

Phosphorescence spectra of DBN consisted of emission due to the exciton ${ }^{17}$ and at least five trap states with spectroscopic trap depths of $28 \mathrm{~cm}^{-1}$ (Trap I), 38 $\mathrm{cm}^{-1}$ (Trap II), $67 \mathrm{~cm}^{-1}$ (Trap III), $120 \mathrm{~cm}^{-1}$ (Trap IV), and $146 \mathrm{~cm}^{-1}$ (Trap V). ${ }^{20}$ These observations were identical to those previously reported, ${ }^{20}$ with the exception of the $120 \mathrm{~cm}^{-1}$ trap. Above $\sim 15^{\circ} \mathrm{K}$ only exciton emission is observed. As the temperature is lowered, the intensity of this emission increases, and trap emission appears. At $\sim 9^{\circ} \mathrm{K}$ the spectrum consists of emission due to the exciton, Traps IV and V. At $4.2^{\circ} \mathrm{K}$, Trap III is also seen in emission, and at $3^{\circ} \mathrm{K}$ emission from all five traps is observed. At $2^{\circ} \mathrm{K}$ the most intense lines are those due to the two shallowest traps ( $I$ and II) and the exciton, the other trap emissions having become weaker. In all the above spectra, the characteristic vibronic progression was observed.

\section{RESULTS AND DISCUSSIONS}

\section{A. Kinetic model for a coupled system of one trap and one-dimensional band}

In order to obtain the form of the phosphorescence response to a short laser pulse, one can consider the following simple kinetic model for a system consisting of a single trap species and an exciton band. ${ }^{21}$ The band and trap levels are connected by the rate constants $K_{i}$ and $K_{0}$, and exhibit decay to the ground state through radiative and nonradiative processes with rate constants $K_{t}$ and $K_{e}$ (see Fig. 2).

The rate constant $\left(K_{0}\right)$ for phonon-assisted detrapping can be described using the model of Fayer and Harris. ${ }^{9,13}$ According to this model, the magnitude of $K_{0}$ at a given temperature will be proportional to the square of the matrix element of the parts of the Hamiltonian responsible for the interaction of the trapped excitation with the lattice vibrations $\left(H_{T P}\right)$ and decay of the resulting intermediate into some linear combination of delocalized band states $\left(H_{i e}\right)$, weighted by the exciton density of states at the energy of the intermediate $\rho_{e}\left(E_{i}\right) . K_{0}$ will also reflect the number of phonons present in the crystal with sufficient energy to mediate the formation of the intermediate state. This phonon distribution will be given by the Planck distribution weighted by the phonon density of states:

$$
\langle n(\epsilon)\rangle=\rho_{p}(\epsilon) /[\exp (\epsilon / k T)-1] .
$$

The resulting expression must be integrated over all phonon energies greater than or equal to $E_{i}$, the energy of the intermediate, and over the values of $E_{i}$ coincident with the exciton band to give the following expression for $K_{0}$ :

$$
\begin{aligned}
K_{0}=\frac{2 \pi}{\hbar} & \int d \epsilon \rho_{p}(\epsilon)\{1 /[\exp (\epsilon / k T)-1]\} \int d E_{i} \rho_{e}\left(E_{i}\right) \\
& \times\left|\left\langle\tau, P\left|H_{\tau P}\right| i, P^{\prime}\right\rangle\left\langle i, P^{\prime}\left|H_{i e}\right| e, P^{\prime}\right\rangle\right|^{2},
\end{aligned}
$$

where the states involved are the trapped excitation 
KINETICS OF BAND-TRAP INTERACTIONS

TCB CRYSTAL

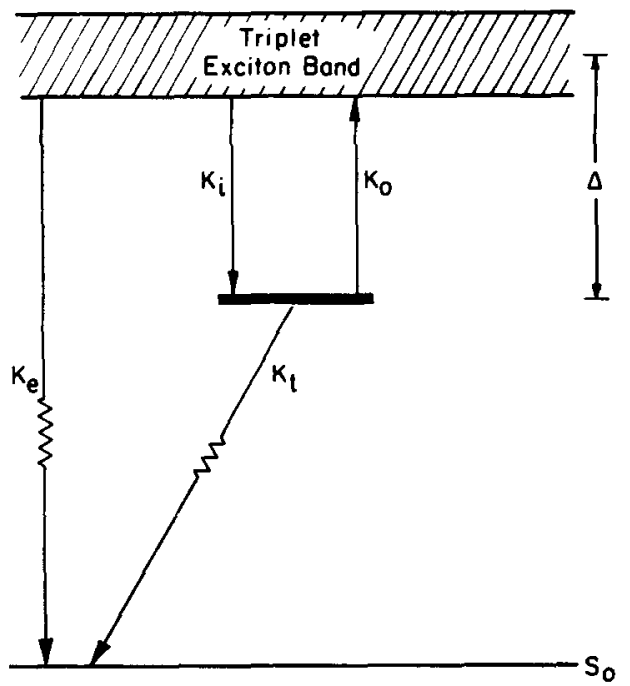

FIG. 2. Kinetic model for a coupled system of one trap and a one-dimensional triplet exciton band. $K_{1}$ and $K_{0}$ are the trapping and detrapping rate constants, respectively, while $K_{e}$ and $K_{t}$ are rate constants for decay to the ground state of the exciton and trap levels, respectively.

plus a phonon state of energy $\epsilon,|\tau, P(\epsilon)\rangle$, the intermediate state of energy $E_{i}$ plus a phonon of energy $\epsilon-E_{i},\left|i, P^{\prime}\left(\epsilon-E_{i}\right)\right\rangle$, and the linear combination of exciton states which results from the decay of the intermediate, $\left|e, P^{\prime}\right\rangle$. This expression, which includes the interaction of the trap with single phonons only, was obtained from the Golden rule by summing over all phonons and intermediate states. At low enough temperatures, if the linear portion of the acoustic phonon branch is populated so that $\rho_{p}(\epsilon)$ is essentially constant, and if $E_{i} \gg k T$, we can write $\langle n(\epsilon)\rangle \propto \exp (-\epsilon / k T)$. For intermediates which contribute significantly to $K_{0}$ and cover a small energy range compared to the trap depth, one obtains the simple expression for the detrapping rate constant,

$$
K_{0}=C \rho_{\theta}\left(E_{i}\right) \exp \left(-E_{i} / k T\right),
$$

where $C$ is a temperature independent constant. This result would be obtained, for example, if $\rho_{e}(E)$ is sharply peaked at some energy $E_{i}$, if the exciton bandwidth is very narrow compared to the trap depth, or in the case of one-dimensional excitons with a peak in $\rho_{e}(E)$ at the bottom of the band if the band is wide compared to $k T$. The above equation indicates that $K_{0}$ will vary radically in the low temperature region ( $T$ $\left.\sim 1^{\circ}-10^{\circ} \mathrm{K}\right)$ for trap depths on the order of tens of reciprocal centimeters. Thus, a marked effect on the phosphorescence response as a function of temperature is expected.

It should be added that one can think of the processes diagrammed in Fig. 2 as occurring among the population of a "single" excited state in the temperature range of interest if the trapping, detrapping, and migration processes occur among the molecules in the one-dimensional chains so that they are spin independent.
A complete description for the dynamics of the coupled trap-exciton system can be obtained from the simplified kinetic equation for $N_{c}$ and $N_{t}$, the number of excitations in exciton and trap levels:

$$
\begin{aligned}
& \dot{N}_{e}(t)=-\left(K_{i}+K_{e}\right) N_{e}(t)+K_{0} N_{t}(t), \\
& \dot{N}_{t}(t)=-\left(K_{t}+K_{0}\right) N_{t}(t)+K_{i} N_{e}(t) .
\end{aligned}
$$

In writing these equations, the excited state concentration is taken to be sufficiently small that nonlinear effects such as biexcitonic annihilation ${ }^{22}$ and effects such as "filling up" of the traps (i.e., the traps are already filled so that the incoming exciton is not welcomed) can be ignored. The solutions for these equations are of the form

$$
\begin{aligned}
& N_{\epsilon}(t)=N_{e}^{+} \exp \left(\alpha_{+} t\right)+N_{e}^{-} \exp \left(\alpha_{-} t\right), \\
& N_{t}(t)=N_{t}^{+} \exp \left(\alpha_{+} t\right)+N_{t}^{-} \exp \left(\alpha_{-} t\right),
\end{aligned}
$$

where

$$
\begin{aligned}
\alpha_{ \pm}=\{1 / 2\}\left\{-\left(K_{0}\right.\right. & \left.+K_{i}+K_{t}+K_{e}\right) \pm\left[\left(K_{i}+K_{e}-K_{t}\right)^{2}\right. \\
& \left.\left.+K_{0}^{2}+2 K_{0}\left(K_{i}-K_{e}+K_{t}\right)\right]^{1 / 2}\right\}
\end{aligned}
$$

and $N_{e}^{+}, N_{e}^{-}, N_{t}^{+}$, and $N_{t}^{-}$are determined by the initial conditions. The initial conditions of interest for the experiments to be described are $N_{a}(t=0)=N_{0}$ and $N_{t}(t=0)=0$, which results in the following expressions for the populations as a function of time:

$$
\begin{aligned}
N_{e}(t)=N_{0}\left\{\left[\left(\alpha_{-}+K_{i}+K_{\theta}\right) /\left(\alpha_{-}-\alpha_{+}\right)\right] \exp \left(\alpha_{+} t\right)\right. \\
\left.+\left[\left(\alpha_{+}+K_{i}+K_{e}\right) /\left(\alpha_{+}-\alpha_{-}\right)\right] \exp \left(\alpha_{-} t\right)\right\}, \\
N_{t}(t)=\left[\left(K_{i} N_{0}\right) /\left(\alpha_{+}-\alpha_{-}\right)\right]\left[\exp \left(\alpha_{+} t\right)-\exp \left(\alpha_{-} t\right)\right] .
\end{aligned}
$$

In order to predict the kinetics of energy transfer, it is useful to graph the decay constants and pre-exponential factors resulting from solution of the above equations as a function of $K_{0}$ for various values of the other parameters. From Figs. 3-5, one observes the following behavior. The exciton population decays as a biexponential. It is dominated in the slow-detrapping regime by the fast $\left[\alpha_{-} \approx-\left(K_{i}+K_{e}\right)\right]$ component due to the combined effects of trapping and decay to the ground state, but still exhibits a long $\left(\alpha_{+} \approx-K_{t}\right)$ tail of lesser magnitude as a result of some small detrapping rate. In this regime the trap builds up as the exciton decays and then decays with its characteristic decay constant $K_{t}$, as should be noted from Eq. (16). In the fast-detrapping regime the triplet population spends little time in the trap, and the decay of the exciton is dominated by the slow component $\left(\alpha_{+} \approx-K_{e}\right)$ at the decay rate to the ground state characteristic of the exciton. The trap population is minimal. It builds up very fast $\left(\alpha_{-} \approx-K_{0}\right)$ and then decays with a rate equal to that of the exciton decay owing to the rapid communication with the exciton band.

This model has been presented for the simplest case of a single trap. It should, however, be equally applicable to systems with multiple trap species, although a cubic or higher order equation for the decay constants will result in general. While this is not particularly formidable, extraction of the parameters $K_{i}, K_{0}$, etc. , from experimental data may be more difficult and 
DEPENDENCE OF THE BUILD-UP IN TRAP POPULATION ON TRAPPING AND DETRAPPING RATE CONSTANTS. TCB CRYSTAL

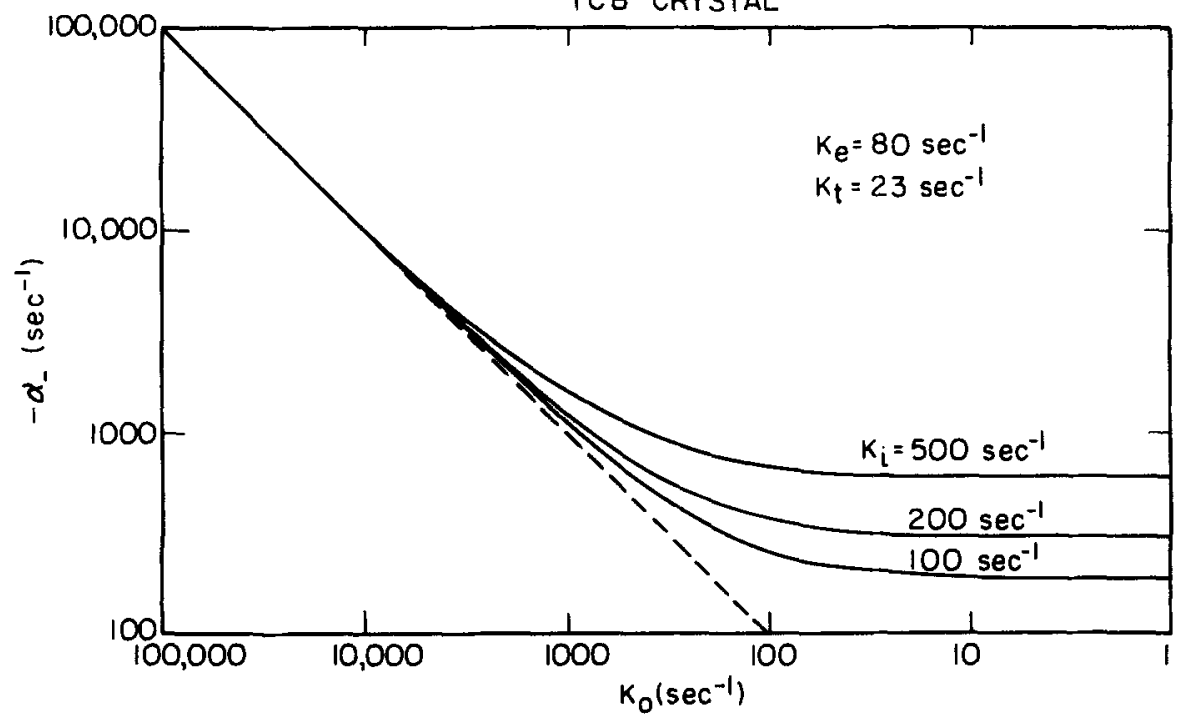

FIG. 3. Prediction of the kinetic model for the variation of $\alpha_{-}$(the fast exciton decay component and trap build-up rate) with detrapping rate constant. The curves correspond to different values of $K_{i}$, the trapping rate constant.

ambiguous.

One can also use this simple model to calculate the expected steady-state populations under conditons of continuous illumination. If population is fed to the exciton band with constant rate $R$, the kinetic equations in the steady state become

$$
\begin{aligned}
& \dot{N}_{e}=0=R-N_{e}\left(K_{e}+K_{i}\right)+N_{t}\left(K_{0}\right), \\
& \dot{N}_{t}=0=-N_{t}\left(K_{0}+K_{t}\right)+N_{e}\left(K_{i}\right) .
\end{aligned}
$$

The solutions are given by

$$
N_{t}=K_{i} R /\left(K_{e} K_{0}+K_{e} K_{t}+K_{i} K_{t}\right)
$$

and

$$
N_{e}=\left(K_{0}+K_{t}\right) R /\left(K_{e} K_{0}+K_{e} K_{t}+K_{i} K_{t}\right) .
$$

A useful ratio which gives the fraction of trap population is

$$
N_{t} /\left(N_{e}+N_{t}\right)=1 /\left[1+\left(K_{0} / K_{i}\right)+\left(K_{t} / K_{i}\right)\right] .
$$

In the Boltzmann regime, this ratio will be determined by the one excitation partition function ${ }^{9}$ of the system (single trap and $N$ exciton levels), i.e.,

$$
N_{t} /\left(N_{e}+N_{t}\right)=1 / Z
$$

and

$$
Z=1+\sum_{k} \exp [-E(k) / k T],
$$

where the zero of the energy is taken at the trap energy. If $\Delta$ is the trap depth below the $k=0$ level of a one-dimensional band [i.e., $E(k)$ takes a very simple form; see Eq. (1)], the partition function takes the form

$$
\begin{aligned}
Z= & 1+\exp (-\Delta / k T) \\
& +\sum_{k=\pi / a n}^{(n-1)(\pi / a n)} 2 \exp \{[-\Delta-2 \beta(1-\cos k a)] / k T\}
\end{aligned}
$$

DEPENDENCE OF THE TRAP DECAY ON TRAPPING AND DETRAPPING RATE CONSTANTS.

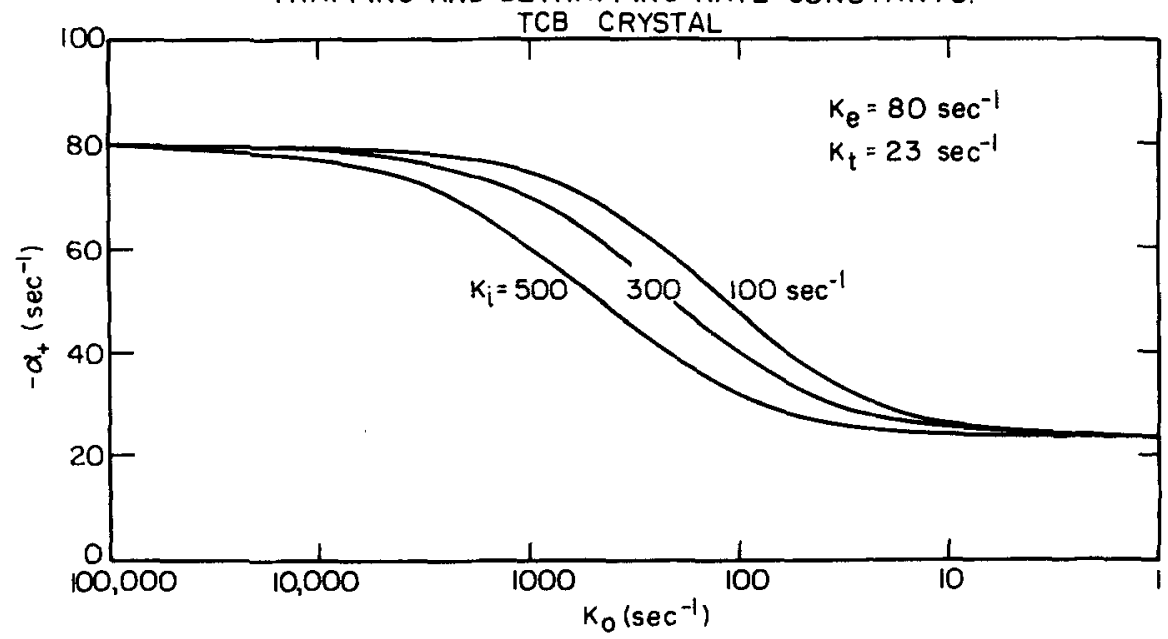

FIG. 4. Prediction of the kinetic model for the variation of $\alpha_{+}$(exciton and trap decay rate) with the detrapping rate constant for different values of the trapping rate constant. 


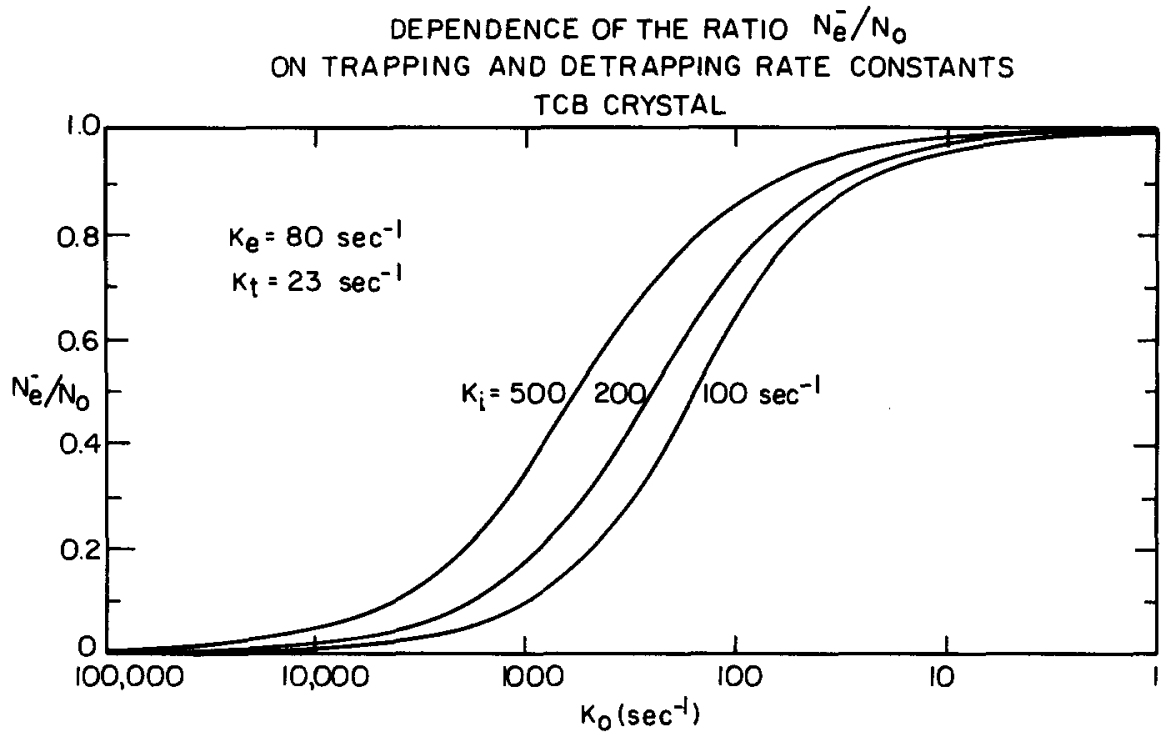

FIG. 5. Prediction of the kinetic model for the magnitude of the fast component of the exciton decay compared with the initial exciton population $N_{0}$. The functional dependence of the ratio $N_{e}^{-} / N_{0}$ on the detrapping rate constant is displayed for different values of the trapping rate constant.

for the $2 n$ states of the band. The $k=0$ partition term is $\exp (-\Delta / k T)$, and the total number of molecules is therefore $(2 n+1)$. This result [Eqs. (22) and (24)] is to be compared with Eq. (21) under the condition necessary to produce thermal equilibrium, namely that the excitation sample band and trap states with sufficient rapidity that equilibrium is attained during its lifetime. In other words, $K_{0}$ and $K_{i} \gg K_{t}$ and $K_{e}$, and therefore

$$
K_{0} / K_{i} \gg K_{t} / K_{i}
$$

As noted in Eq. (9), $K_{0}$ can be approximated as

$$
K_{0} \propto N \exp (-\Delta / k T),
$$

where the density of exciton states is replaced by the number of exciton levels, $N$. If the system is in Boltzmann equilibrium, it must be that $K_{0} / K_{i}=N_{e} / N_{t}$ $=N \exp (-\Delta / k T)$. Thus, Eq. (21) becomes

$$
N_{t} /\left(N_{e}+N_{t}\right)=1 /[1+N \exp (-\Delta / k T)],
$$

which is equivalent to $1 / Z$ [see Eq. (23)] if the bandwidth is small compared to the trap depth, i. e., $4 \beta$ $\ll \Delta$. In fact, the parameters of the model do not include the bandwidth, and $k$-dependent processes are not considered. It is therefore expected to be most applicable in the narrow band and/or large trap depth limits.

\section{B. Experimental determination of the kinetic parameters}

\section{1, 4-dibromonaphthalene}

Pulse excitation-time resolved phosphorescence measurements of build-up and decay rates for the trap states in DBN were made at several temperatures from $2^{\circ} \mathrm{K}$ to $9^{\circ} \mathrm{K}$. The results of these measurements using an excitation wavelength of $4789 \AA\left(688 \mathrm{~cm}^{-1}\right.$ above the exciton origin) are shown in Fig. 6 .

The behavior of the phosphorescence response from some of the traps qualitatively conforms to that predicted by our kinetic model. At $2^{\circ} \mathrm{K}$, Traps I, II, and IV all exhibit phosphorescence which builds up at the exciton decay rate and decays at the trap decay rate.
At $3^{\circ} \mathrm{K}$ similiar behavior is observed, except for Trap $\mathrm{I}$, which has shortened decay rate. At $4.2^{\circ} \mathrm{K}$, detrapping from Traps I and II is sufficiently rapid that the build-up rate is extremely fast and the decay rate is that of the exciton, indicating that complete equilibration of the traps and band has taken place. How-

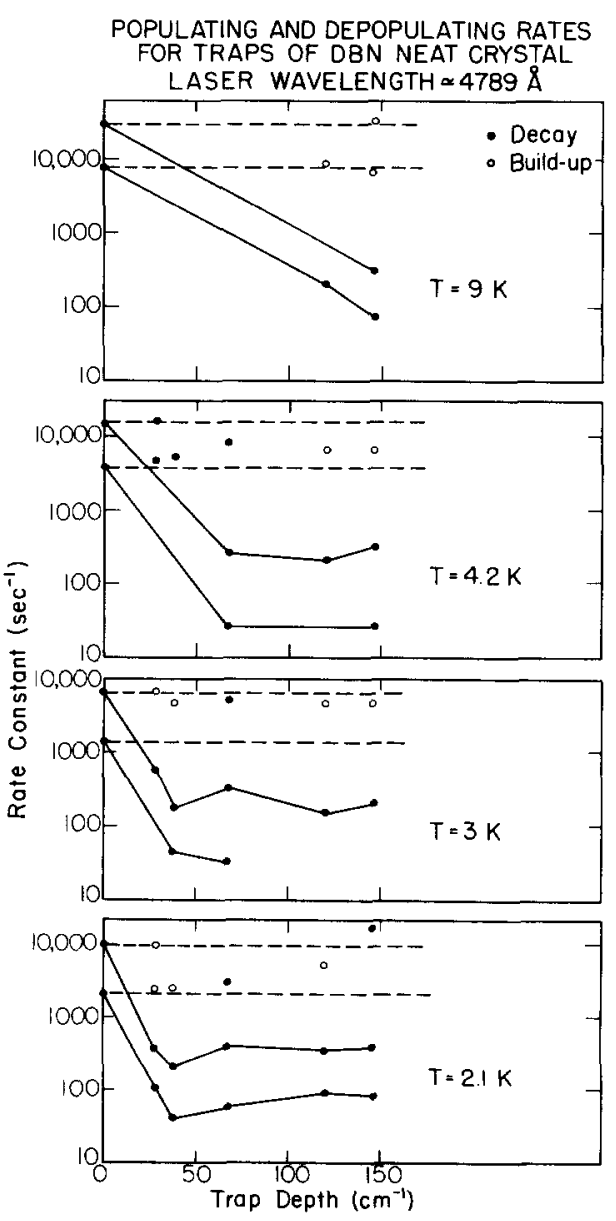

FIG. 6. This figure shows the build-up (circles) and decay (dots) rates for the exciton and trap populations in neat 1,4dibromonaphthalene as a function of trap depth at four different temperatures. The excitation wavelength was $4789 \AA$. 
ever, the detrapping rate from Trap IV is not sufficient to produce similar behavior either at $4.2^{\circ} \mathrm{K}$ or at $9^{\circ} \mathrm{K}$, and the trap build-up rate is equal to the exciton decay rate. It seems likely that these features of the bandtrap equilibria could be understood on the basis of the kinetics of band-trap interactions with themselves and with the ground state of the crystal.

However, there are some difficulties with the interpretation of certain other qualitative features. A buildup of Trap III phosphorescence was not observed at any temperature. Population appeared very quickly (within $1 \mu \mathrm{sec}$ ), and three decay components were observed, one with a decay rate similar to the exciton, and two with decay rates similar to those of other traps. On the other hand, Trap V seems to behave as expected except at $2^{\circ} \mathrm{K}$, where the buildup becomes very fast, in contrast to its behavior at higher temperatures. Thus, the extraction of specific information relating the exciton dynamics to the observed phosphorescence response is difficult owing to the increased complexity of the DBN band-trap system compared to TCB. However, the observed transient and steady state phosphorescence behavior clearly shows that communication between the exciton band and the various traps does exist, and therefore the interpretation of other types of measurements designed to probe exciton dynamics must include these interactions to fully understand the problem. In particular, if the triplet exciton ESR spectrum ${ }^{23-25}$ obtained by modulation techniques were measured in a temperature range where the detrapping rate is sufficiently large that band-trap equilibrium is attained on a time scale which is shorter than the period of the modulation, the effect of this band-trap equilibrium must be explicitly considered. These conditions can result in the observation of trap ESR transitions in the exciton spectrum even when the modulation period is shorter than the trap lifetime. ${ }^{26}$ All that is necessary is that equilibrium be reached during the modulation period and that a significant fraction of the total population be involved. As our measurements have shown, trapband communication may take place within the exciton lifetime at some temperatures (see Fig. 6). We should add that the above discussions represent only a concern, since all that we know at this point (with certainty) is that there is effective communication between band and trap states on a short time scale. The complexity of the multiple trap sites in this syste $\mathrm{m}^{20}$ does not allow an unequivocal demonstration of coherence. Such is not the case, however, with the simpler TCB system.

\section{1, 2, 4, 5-tetrachlorobenzene}

Direct laser excitation of the triplet exciton origin in TCB resulted in the exciton and $x$-trap phosphorescence response shown in Figs. 7 and 8 for temperatures of $1.25^{\circ}$ and $4.2^{\circ} \mathrm{K}$. The system clearly exhibits the general features of our kinetic model for a coupled band-trap system. At $4.2^{\circ} \mathrm{K}$, phonon-assisted promotion (detrapping) of a trapped excitation to the exciton band is rapid; both exciton and trap populations decay at a rate which is characteristic of the exciton, and the buildup of the trap phosphorescence is very fast. In the slow-detrapping regime $\left(1.25^{\circ} \mathrm{K}\right)$, the trap population builds up as the exciton decays, and it then decays with a rate constant characteristic of the trap excited triplet lifetime. The exciton decay rate reflects the exciton lifetime and the trapping rate.

Such measurements have been made over a range of temperatures, and the time-resolved phosphorescence decay was decomposed into a sum of exponentials. Build-up and decay rates as well as the relative intensity of each exponential component were then obtained as a function of temperature. These data for the TCB neat crystal system are shown in Tables I, II, and III. The rate constants are listed as a function of temperature for excitation wavelengths corresponding to the exciton origin and two shorter wavelengths. By re-

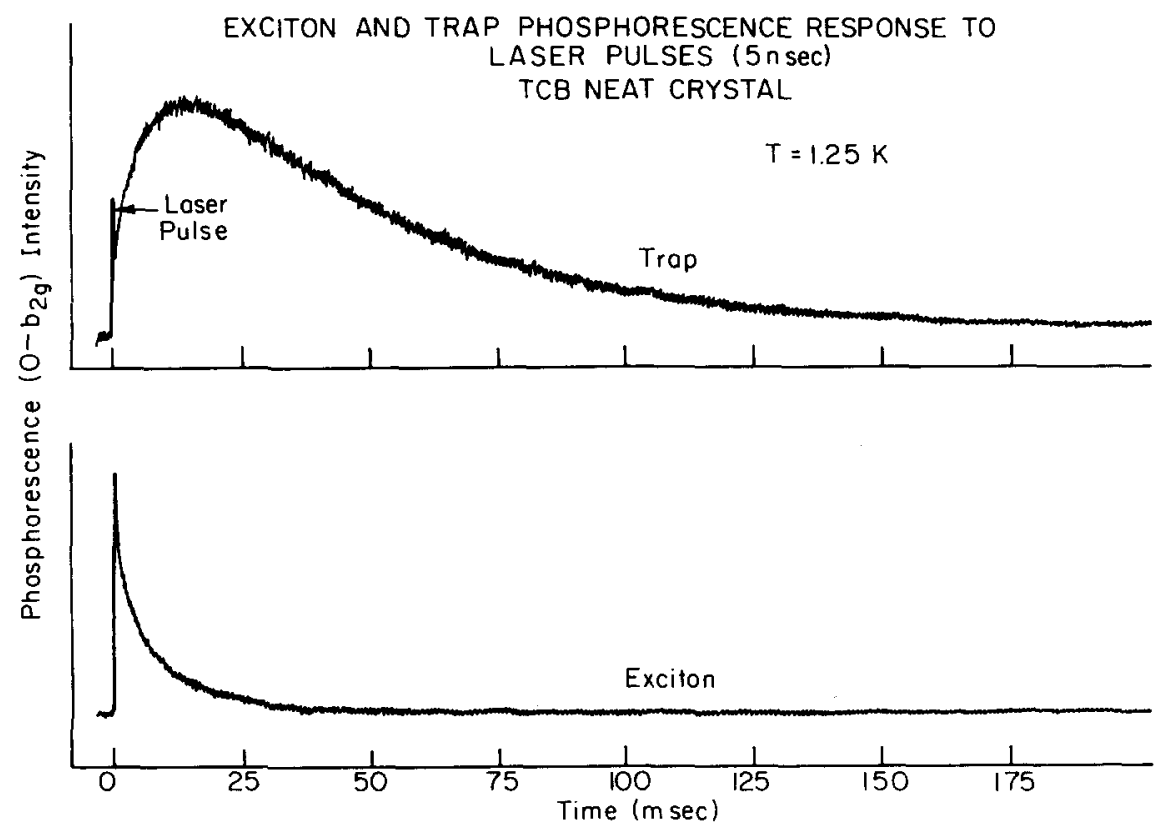

FIG. 7. Phosphorescence response of neat $\mathrm{TCB}$ to pulsed laser excitation in the low temperature-slow detrapping regime. Note that the exciton decay and trap build-up rates are similar. 


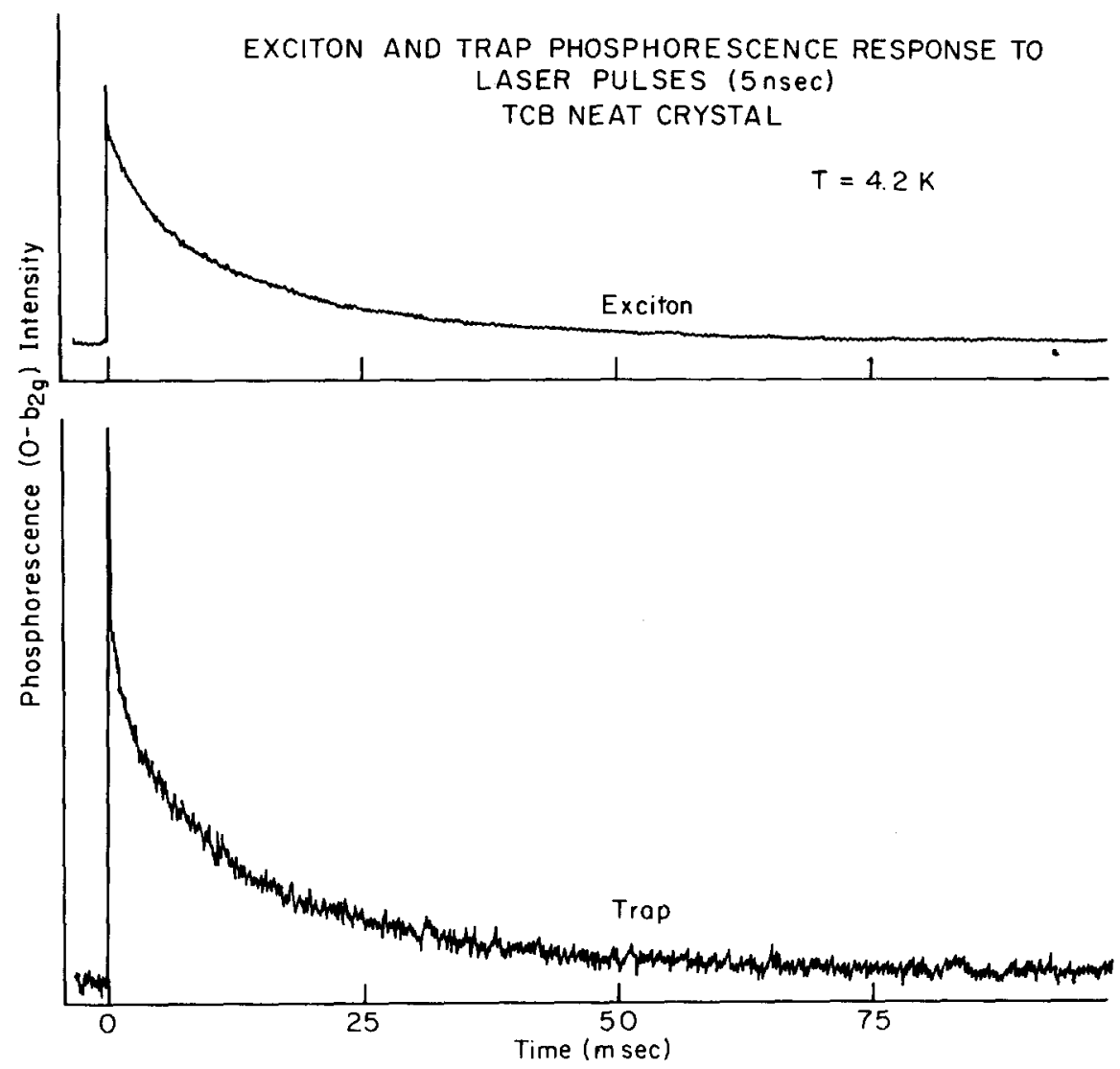

FIG. 8. Phosphorescence response of neat $\mathrm{TCB}$ to pulsed laser excitation in the high temperature-fast detrapping regime. Note that the exciton and trap decay at the same rate and no trap population buildup is observed on this time scale.

lating this temperature dependence to that predicted by the model, the rate constants, $K_{0}, K_{i}, K_{e}$, and $K_{t}$, can be determined and related to the energy transfer dynamics in the crystal.

The fitting of the calculated temperature dependence to the experimental data was approached from two directions. First, $K_{e}$ and $K_{t}$ were estimated from the high and low temperature limits of the trap and/or exciton decay rates. $K_{i}$ was estimated from the trap build-up rate in the low temperature limit. A curve for $N_{e}^{-}$as a function of $K_{0}$ was then calculated, and the value of $K_{0}$ corresponding to each temperature was found by matching the experimental values of $N_{e}^{-}$to this curve. A plot of $\ln K_{0}$ vs $1 / T$ then gives $\Delta E$, the effective energy difference between the localized trap state and the states to which detrapping is taking place. Also this allows calculation of $\alpha_{+}$and $\alpha_{-}$to be made and compared with the experimental data. The various parameters are then adjusted for the best overall fit. This procedure gives $K_{e}=80 \mathrm{sec}^{-1}, K_{t}=23 \mathrm{sec}^{-1}, K_{i}$ $=250 \mathrm{sec}^{-1}$, and $\Delta E=15 \mathrm{~cm}^{-1}$. The fits to the experimental data and the plot of $K_{0}$ vs $1 / T$ are shown in Figs. 9-11 (solid curves).

The second approach to fitting the data was based on the fact that the trap build-up rate $\alpha$. approaches the detrapping rate constant $K_{0}$ in the high temperature limit. Thus, a straight line through this region of the experimentally determined values of $\alpha_{-}$directly gives $K_{0}$ vs $1 / T$ and a value of $\Delta E=20 \mathrm{~cm}^{-1}$. The best fit to $N_{e}^{\circ}$ and $\alpha_{-}$is obtained with $K=80 \mathrm{sec}^{-1}, K_{t}=23 \mathrm{sec}^{-1}$, and $K_{i}=150 \mathrm{sec}^{-1}$ (see Fig. 11, dotted curve).
TABLE I. Phosphorescence build-up and decay rate constants for neat TCB: excitation wavelength $=3748 \AA$ [exciton origin $(0,0)]$.

\begin{tabular}{|c|c|c|c|c|c|}
\hline $\begin{array}{l}\text { Temperature } \\
\left({ }^{\circ} \mathbf{K}\right)\end{array}$ & $\begin{array}{r}\text { Exciton d } \\
b_{2} \text { origi }\end{array}$ & $\begin{array}{l}\text { cay rates } \\
\left(\sec ^{-1}\right)^{2}\end{array}$ & $\begin{array}{l}x \text { Trap }\left(b_{2 g} \text { origin) }\right. \\
\text { buildup }\left(\mathrm{sec}^{-t}\right)\end{array}$ & Decay & $\left.\sec ^{-1}\right)^{2}$ \\
\hline 1.25 & $\begin{array}{l}104 \\
884\end{array}$ & $\begin{array}{l}\text { (1) } \\
(0.69)\end{array}$ & 128 & 22.5 & \\
\hline 1.50 & $\begin{array}{l}23.3 \\
166\end{array}$ & $\begin{array}{l}(0.09) \\
(1)\end{array}$ & 204 & 25.2 & \\
\hline 1.75 & $\begin{array}{l}16.0 \\
216\end{array}$ & $\begin{array}{l}(0.05) \\
(1)\end{array}$ & 254 & 25.8 & \\
\hline 2.00 & $\begin{array}{l}17.8 \\
283\end{array}$ & $\begin{array}{l}(0.10) \\
(1)\end{array}$ & 354 & 26.6 & \\
\hline 2.25 & $\begin{array}{l}17.3 \\
62.9 \\
476\end{array}$ & $\begin{array}{l}(0.34) \\
(0.25) \\
(1)\end{array}$ & 453 & $\begin{array}{l}13.5 \\
44.7\end{array}$ & $\begin{array}{l}(0.53) \\
(1)\end{array}$ \\
\hline 2.50 & $\begin{array}{l}265 \\
843\end{array}$ & $\begin{array}{l}(1) \\
(0.71)\end{array}$ & 1480 & $\begin{array}{l}18.2 \\
47.3\end{array}$ & $\begin{array}{l}(0.46) \\
\text { (1) }\end{array}$ \\
\hline 2.75 & $\begin{array}{r}20.3 \\
53.8 \\
1180\end{array}$ & $\begin{array}{l}(0.97) \\
(1) \\
(0.76)\end{array}$ & 4123 & 33.7 & \\
\hline 3.00 & $\begin{array}{l}23.9 \\
65.1\end{array}$ & $\begin{array}{l}\text { (1) } \\
\text { (1) }\end{array}$ & $\cdots$ & $\begin{array}{l}26.7 \\
106\end{array}$ & $\begin{array}{l}(1) \\
(0.89)\end{array}$ \\
\hline 3.25 & $\begin{array}{l}23.5 \\
71.4\end{array}$ & $\begin{array}{l}(0.69) \\
(1)\end{array}$ & $\ldots$ & $\begin{array}{l}19.4 \\
79.0\end{array}$ & $\begin{array}{l}(0.5) \\
(1)\end{array}$ \\
\hline 3.50 & $\begin{array}{l}22.5 \\
70\end{array}$ & $\begin{array}{l}(0.59) \\
(1)\end{array}$ & $\ldots$ & $\begin{array}{c}26.3 \\
137\end{array}$ & $\begin{array}{l}(0.8) \\
(1)\end{array}$ \\
\hline 3.75 & $\begin{array}{l}36.7 \\
135\end{array}$ & $\begin{array}{l}\text { (1) } \\
(0.78)\end{array}$ & $\ldots$ & $\begin{array}{l}33.7 \\
169\end{array}$ & $\begin{array}{l}(0.72) \\
(1)\end{array}$ \\
\hline 4.00 & $\begin{array}{l}37.9 \\
128\end{array}$ & $\begin{array}{l}\text { (1) } \\
(0.91)\end{array}$ & $\cdots$ & 51.9 & \\
\hline 4.20 & $\begin{array}{c}35.2 \\
158\end{array}$ & $\begin{array}{l}(0.72) \\
(1)\end{array}$ & $\cdots$ & $\begin{array}{r}46.7 \\
239\end{array}$ & $\begin{array}{l}\text { (1) } \\
(0.87)\end{array}$ \\
\hline
\end{tabular}

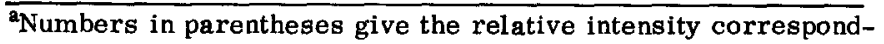
ing to each decay component (pre-exponential factor). 
TABLE II. Phosphorescence decay and build-up rate constants for neat TCB: excitation wavelength $=3733 \AA\left[108 \mathrm{~cm}^{-1}\right.$ above exciton $\left.(0,0)\right]$.

\begin{tabular}{|c|c|c|c|c|c|c|c|c|c|}
\hline \multirow{5}{*}{$\begin{array}{l}\text { Temperature } \\
\left({ }^{\circ} \mathrm{K}\right)\end{array}$} & & & & & \multicolumn{5}{|c|}{$x$-trap rates $\left(\mathrm{sec}^{-1}\right)$} \\
\hline & \multicolumn{4}{|c|}{ Exciton decay rates $\left(\sec ^{-1}\right)^{a}$} & \multicolumn{2}{|c|}{$b_{2 g}$ Origin } & \multicolumn{3}{|c|}{$(0,0)$ Origin } \\
\hline & \multicolumn{2}{|c|}{$b_{2 g}$ Origin } & \multirow[t]{2}{*}{$(0,0)$} & \multirow[t]{3}{*}{ Origin } & \multirow[t]{2}{*}{ Buildup } & \multirow[t]{3}{*}{$\operatorname{Decay}^{2}\left(\sec ^{-1}\right)$} & \multirow[t]{3}{*}{ Buildup } & \multicolumn{2}{|c|}{$\operatorname{Decay}^{\mathrm{a}}\left(\mathrm{sec}^{-1}\right)$} \\
\hline & 255 & $(0.36)$ & & & & & & & \\
\hline & 990 & (1) & & & & & & & \\
\hline \multirow[t]{2}{*}{10.45} & 103 & $(0.29)$ & & & & & & & \\
\hline & 467 & (1) & & & & & & & \\
\hline \multirow[t]{2}{*}{8.0} & 99.8 & (1) & 439 & & & & & 90.5 & $(0.86)$ \\
\hline & 681 & $(0.69)$ & & & & & & 599 & (1) \\
\hline \multirow[t]{2}{*}{4.2} & 46 & (1) & 42.7 & (1) & & & & 52.3 & \\
\hline & 170 & $(0.13)$ & 265 & $(0.18)$ & & & & & \\
\hline \multirow[t]{3}{*}{2.8} & 26.9 & (1) & 34.7 & (1) & 5540 & 30 & 3090 & 36.2 & \\
\hline & 84.3 & $(0.44)$ & 130 & $(0.34)$ & & & & & \\
\hline & 4000 & $(0.67)$ & & & & & & & \\
\hline \multirow[t]{3}{*}{2.1} & 29.6 & $(0.17)$ & 44.4 & $(0.18)$ & 349 & 33.1 & 378 & 32 & \\
\hline & 406 & (1) & 317 & (1) & & & & & \\
\hline & 6185 & $(0.72)$ & 1855 & $(0.38)$ & & & & & \\
\hline
\end{tabular}

${ }^{2}$ Numbers in parentheses give the relative intensity corresponding to each decay component (preexponential factor).

It must be noted that while the model calculations predict very little variation of $\alpha$. with $1 / T$ in the low temperature limit, the experimental values slowly increase with increasing temperatures. One possible source of this discrepancy is an increasing value of the exciton decay constant $K_{e}$ with temperature. In fact, from Table II one can see that the exciton decay constant(s) does continue to increase with temperature above $4.2^{\circ} \mathrm{K}$ where only exciton emission is observed. This effect could, for example, be the result of a thermally activated nonradiative decay channel. In order to illustrate this effect, Fig. 11 also shows a calculated curve for $\alpha$. using $K_{e}=K_{t}=23 \mathrm{sec}^{-1}$ and $K_{i}$ $=125 \mathrm{sec}^{-1}$. If $K_{e}$ increases with increasing temperature, deviation from the calculated curve in the positive direction is expected until the limit is reached

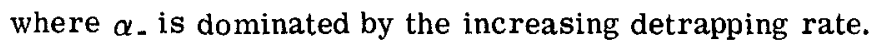

Based on the above considerations, one can set a limit on the trapping rate constant of $K_{i}=100-300 \mathrm{sec}^{-1}$ and on the detrapping energy of $\Delta E=15-20 \mathrm{~cm}^{-1}$. These limits reflect both the uncertainty in our estimates of $K_{i}$ and $\Delta E$ due to the experimental uncertainty in the measurements themselves as well as the "best fit" to our model calculations. For the purpose of this analysis, it was found that the temperature dependence of $K_{0}$ was adequately described by

$$
K_{0} \propto N \exp (-\Delta E / k T) \text {. }
$$

The obtained value of $\Delta E$ is to be compared with a spectroscopically measured trap depth of $\Delta=21.3 \mathrm{~cm}^{-1} .^{16}$

\section{Trapping rate and coherent energy migration: coherence length}

The measurement of the trapping rate constant becomes especially significant once the trap concentration is known. The concentration of $x$ traps in the crystal (i.e., the average number of exciton states per trap) can be approximately determined by assuming that the steady state $x$-trap population obeys Boltzmann statistics and fitting the experimentally determined phosphorescence temperature dependence to a curve obtained from Eqs. (22) and (24). Using an integral approximation to the sum in Eq. (24) and applying the formula (zero-order modified Bessel function of the first kind)

TABLE III. Phosphorescence decay and build-up rate constants for neat TCB: excitation wavelength $=3744 \AA\left[26 \mathrm{~cm}^{-1}\right.$ above exciton $\left.(0,0)\right]$.

\begin{tabular}{|c|c|c|c|c|c|c|c|c|}
\hline \multirow{4}{*}{$\begin{array}{l}\text { Temperature } \\
\left({ }^{\circ} \mathrm{K}\right) \\
4.2\end{array}$} & & & & & \multicolumn{4}{|c|}{$x$-Trap rates $\left(\mathrm{sec}^{-1}\right)$} \\
\hline & \multicolumn{4}{|c|}{ Exciton decay rates $\left(\sec ^{-1}\right)^{a}$} & \multicolumn{2}{|c|}{$b_{2 g}$ Origin } & \multicolumn{2}{|c|}{$(0,0)$ Origin } \\
\hline & \multicolumn{2}{|c|}{$b_{2 g}$ Origin } & \multicolumn{2}{|c|}{$(0,0)$ Origin } & Buildup & Decay $^{2}$ & Buildup & Decay $^{\mathrm{a}}$ \\
\hline & $\begin{array}{l}27.9 \\
75.7\end{array}$ & $\begin{array}{l}(0,62) \\
(1)\end{array}$ & $\begin{array}{l}28.1 \\
79.0\end{array}$ & $\begin{array}{l}(0.57) \\
(1)\end{array}$ & & & & \\
\hline 1.25 & & & $\begin{array}{l}67.5 \\
273\end{array}$ & $\begin{array}{l}(0.86) \\
(1)\end{array}$ & 133 & 20.6 & 128 & 21.2 \\
\hline
\end{tabular}

${ }^{2}$ Numbers in parentheses give the relative intensity corresponding to each decay component (pre-exponential factor). 


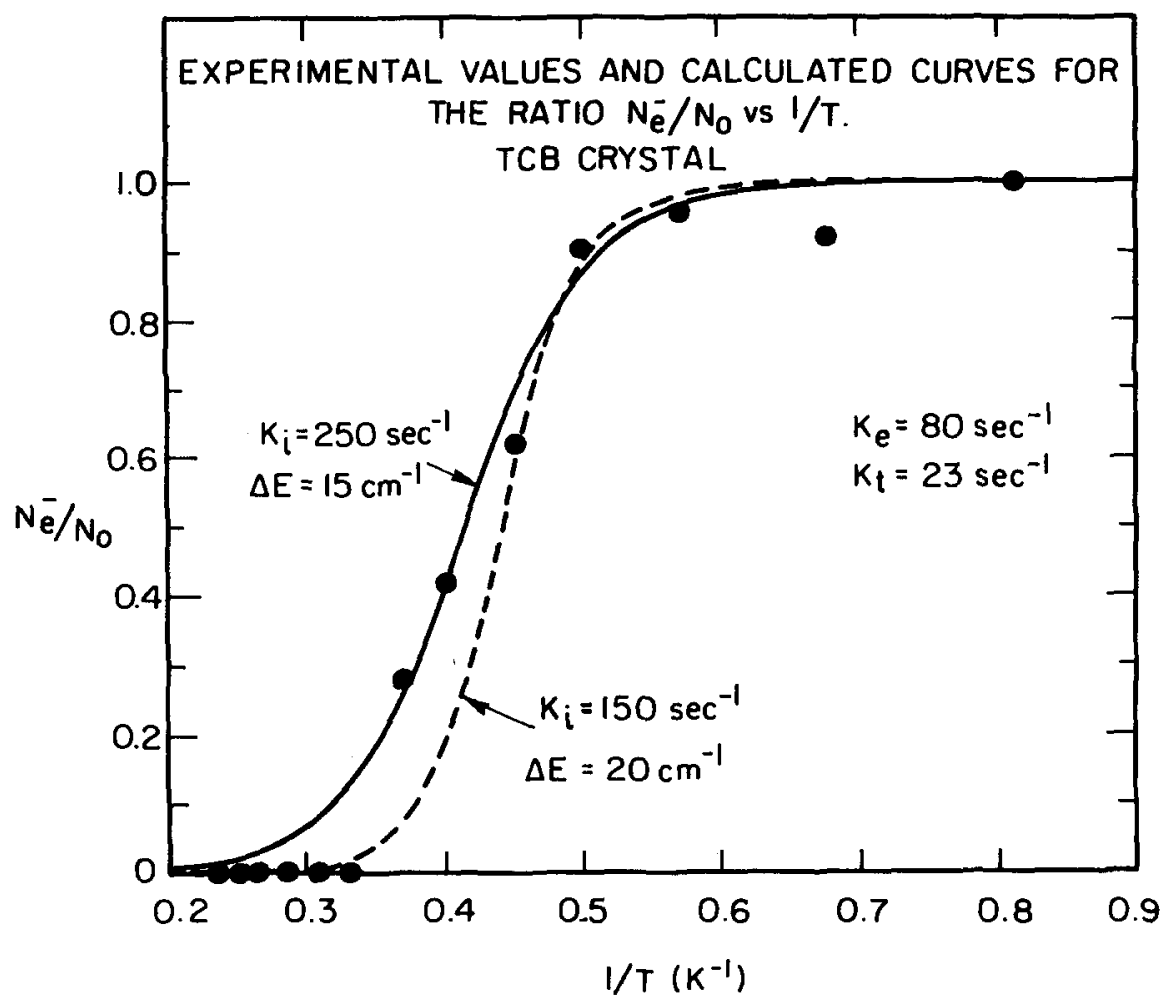

FIG. 9. Calculated fits to experimental data for the ratio $N_{e}^{-} / N_{0}$. The solid curve corresponds to the best fit for $N_{e}^{-} / \Lambda_{0}$, and gives the values of $K_{0}$ vs $1 / T$ shown in Fig. 10. The dotted curve corresponds to values of $K_{0}$ obtained from the plot of $\alpha_{-}$vs $1 / T$ (see Fig, 11).

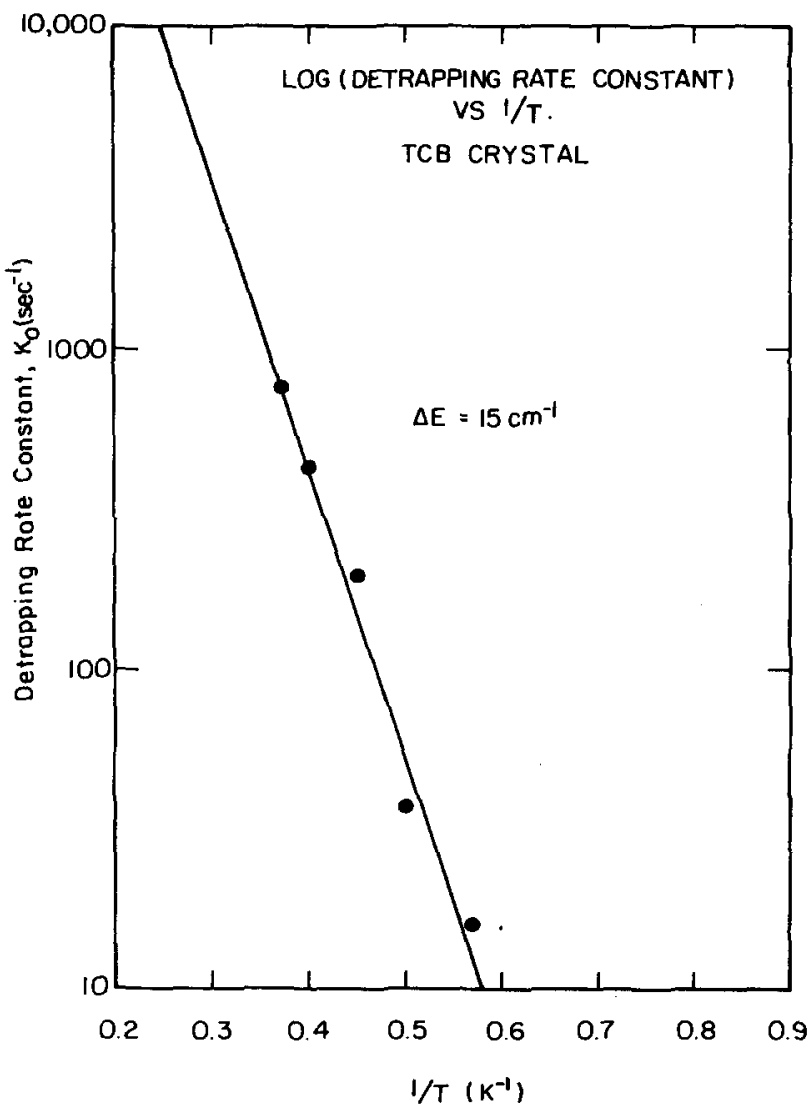

FIG. 10. Detrapping rate constant vs $1 / T$ obtained from the solid curve in Fig. 9 and calculations based on the kinetic model: $\Delta E$ represents the energy difference between the trap level and the intermediate which is assumed to be active in mediating phonon-assisted detrapping.

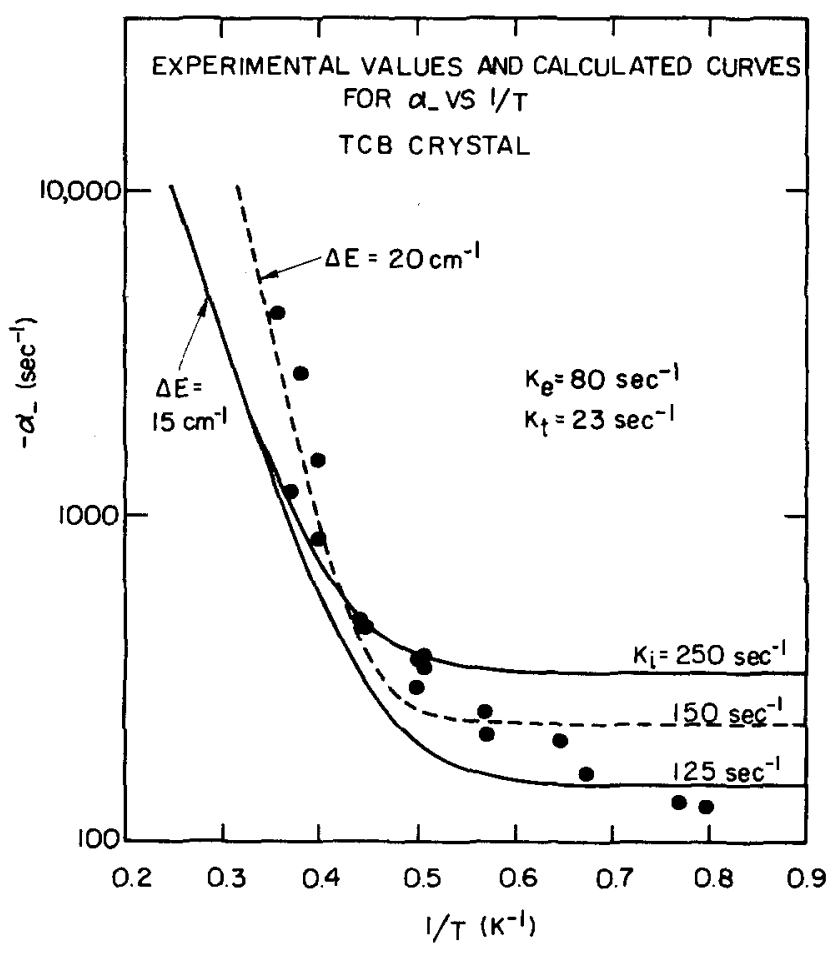

FIG. 11. Calculated fits to the data for $\alpha_{-}$vs $1 / T$. The solid curves were calculated from the model equation using the values of $K_{0}$ vs $1 / T$ from Fig. 10 . The curve for $K_{i}=125 \mathrm{sec}^{-1}$ corresponds to $K_{t}=K_{e}=23 \mathrm{sec}^{-1}$ rather than the values shown in the figure. The dotted curve is the best fit to $\alpha_{-}$, and the corresponding values of $K_{0}$ vs $1 / T$ were used to calculate the dotted curve in Fig. 9. These latter calculations give a value of $\Delta E$ of $20 \mathrm{~cm}^{-1}$. 


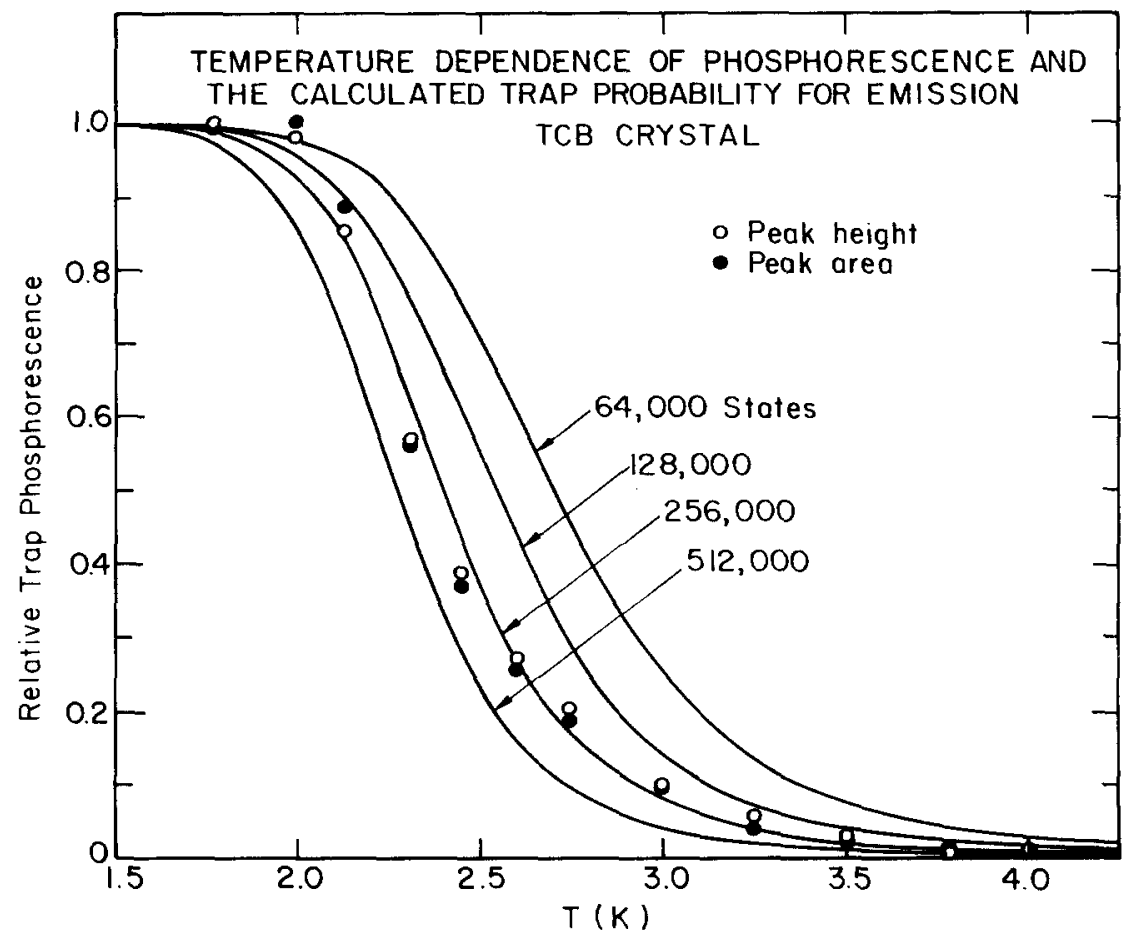

FIG。12. Temperature dependence of steady state phosphorescence emission of the $x$-trap origin in the TCB neat crystal used in these investigations. The circles represent measurements of the recorded height of the peak, while the dots represent the corresponding areas of the peaks. The solid curves were calculated from $\mathrm{Eq}$. (30), for $4 \beta=1 \mathrm{~cm}^{-1}$ and $\Delta$ $=21.3 \mathrm{~cm}^{-1}$. It should be mentioned that the calculated curves are insensitive to $\beta$ in the narrow band limit $(4 \beta \ll \Delta)$ and that the uncertainty in the fit is approximately $25 \%$ which is within our experimental error for $K_{i}$.

$$
I_{0}(\zeta)=(1 / \pi) \int_{0}^{\pi} \exp (\zeta \cos \theta) d \theta,
$$

one obtains an expression for the trap probability (the zero of energy is now taken at the center of the band)

$$
\begin{aligned}
P_{\text {tr } 20} & =\frac{\exp [-(2 \beta-\Delta) / k T]}{\left\{\exp [-(2 \beta-\Delta) / k T]+N I_{0}(2 \beta / k T)\right\}} \\
& \equiv \exp [-(2 \beta-\Delta) / k T](Z)^{-1},
\end{aligned}
$$

where $N$ is the number of exciton states per trap. The experimental data and calculated curves for $\beta=0.25$ $\mathrm{cm}^{-1}$, the various values of $N$, and the tabulated values of $I_{0}$ are shown in Fig. 12. Thus, the chain length is estimated to be 256000 molecules.

These two pieces of information (the $x$-trap concentration and trapping rate constant) can result in increased insight into the coherent nature of exciton propagation in these crystals. An $x$-trap concentration of $1 / 256000$ implies a one-dimensional exciton chain length of $9.6 \times 10^{5} \AA$ on the average. The thermally averaged group velocity, which varies less than $6 \%$ in the temperature range $1-4^{\circ} \mathrm{K}$, can be calculated from the exciton dispersion: $\left\langle V_{g}\right\rangle=2700 \mathrm{~cm} / \mathrm{sec}$ at $1.3^{\circ} \mathrm{K}$. If one takes $K_{i}=200 \mathrm{sec}^{-1}$, the completely coherent limit $\left[\mathrm{Eq}\right.$. (3)] gives $\alpha=7 \times 10^{-4}$ for the trapping probability per excition-trap encounter, a value that seems quite low. On the other hand, the random hopping model [Eq. (4)] estimates a trapping rate constant on the order of $K_{i}=0.46 \mathrm{sec}^{-1}$, which is too small to be reconciled with the experiments. However, in the intermediate regime represented by $\mathrm{Eq}$. (6), one sees that the measurement of $K_{i}$ allows a minimum coherence length to be assigned to the exciton motion; with $K_{1}=200 \mathrm{sec}^{-1}$, one finds that

$$
l \gtrless 700 \AA=186 \text { molecules. }
$$

It should be clear that in the completely incoherent limit [Eq. (4)] the experimental uncertainty in $\beta$ and $d$ will not give a value for $K_{i}$ [Eq. (4)] which is in agreement with the measured value; for a change in $d$ of a factor of 2 (which cannot be the case in our experiments-see Fig. 12), $K_{i}$ will increase by a factor of 4 , which is still much smaller $\left(1.84 \mathrm{sec}^{-1}\right)$ than the reported value. It follows from Eq. (31) and the average group velocity that the minimum coherence time is $\tau_{c} \gtrsim 2.6 \times 10^{-9} \mathrm{sec}$. This coherence time is consistent with the correlation time for scattering obtained from the exciton microwave absorption by Francis and Harris ${ }^{16}$ and that for dimers of proto-TCB in a deutroTCB lattice measured by Zewail and Harris. ${ }^{27-29}$ Using $\tau_{c}=10^{-7} \mathrm{sec}$ (from Ref. 27), one notices that the minimum coherence length reported here is considerably smaller than the maximum value $\left\langle V_{g}\right\rangle \tau_{c}=27000 \AA$. It should be noted [Eq. (6)] that if $l=l_{\max }=27000 \AA$, then as few as $3 \%$ of exciton-trap encounters actually result in trapping of the excitation.

The question of to what extent specific mechanisms determine the coherence of the exciton motion is left unanswered. However, it seems certain that excitonphonon interactions and scattering of the excitons from crystal imperfections other than those responsible for the $x$ traps themselves play important roles. At the lowest temperature $\left(1.15^{\circ} \mathrm{K}\right)$, we observed emission from a trap with an approximate trap depth of $8 \mathrm{~cm}^{-1}$ which has not previously been observed. It seems possible that other similar departures from perfect lattice periodicity perturb the exciton density of states to an extent sufficient to significantly affect the exciton dynamics but do not manifest themselves in the emission spectrum. However, the concentration of disturbed molecules must be relatively high to effectively shorten the coherence length to the extent observed. Hence, exciton-phonon coupling is expected to contribute significantly to the loss of coherence. ${ }^{30}$ 


\section{SUMMARY AND CONCLUSIONS}

We have utilized short light pulses from a tunable dye laser to study the nature of exciton-phonon and exciton-trap interactions in molecular solids $(1,2,4,5-$ tetrachlorobenzene and 1,4-dibromonaphthalene) at different temperatures. The following conclusions can be drawn from these studies:

(1) The time-resolved phosphorescence response reflects the dynamics of exciton-trap equilibria.

(2) In TCB system where the triplet exciton is essentially one dimensional and only one trap is interacting with the band, the dynamics can be described by a simple kinetic model. On the other hand, the presence of multiple "localized" states in the one-dimensional DBN system helped in exploring the many routes of communications between the band and traps which are essential in understanding coherence in this system.

(3) The steady state phosphorescence of TCB $x$ traps indicated the presence of thermal equilibrium with the band state, and thus a chain length of $960000 \AA$ $(\simeq 256000$ molecules $\times 3.76 \AA$ ) was obtained from the temperature dependence of the emission intensity.

(4) The trapping rate constant obtained from the time resolved phosphorescence at different temperatures together with the chain length measurement enabled us to obtain for the first time a minimum coherence length for triplet excitons of molecular solids at low temperatures: $l_{\mathrm{m} 1 \mathrm{n}} \geq 700 \AA=186$ molecules for TCB crystals.

(5) The measured coherence length indicates that exciton-phonon coupling plays an important role in shortening the coherence length for linear chains of very long length.

\section{ACKNOWLEDGMENTS}

This research has been supported in part by the National Science Foundation, a departmental National Science Foundation equipment grant, and in part by the Materials and Molecular Research Division of the Lawrence Berkeley Laboratory under the auspices of the U. S. Energy Research and Development Administration.

${ }^{1}$ A. S. Davydov, Theory of Molecular Excitons (McGraw-Hill, New York, 1962).

${ }^{2}$ T. Holstein, Ann. Phys. (N.Y.) 8, 343 (1959).

${ }^{3}$ M. Grover and R. Silbey, J. Chem. Phys, 54, 4843 (1971).

${ }^{4}$ H. Haken and G. Strobl, in The Triplet State, edited by A. B. Zahlan (Cambridge U. P., Cambridge, England, 1967); P. Reineker and H. Haken, Z. Phys, 256, 300 (1972).

${ }^{5}$ C. B. Harris and M. D. Fayer, Phys. Rev. B 10, 1784 (1974).
${ }^{6} \mathrm{~S}$. Rice in The Triplet State, edited by A. B. Zahlan (Cambridge U. P. , Cambridge, England, 1967).

${ }^{7}$ A. H. Francis and C. B. Harris, Chem. Phys, Lett. 9, 181 (1971).

${ }^{8} \mathrm{~V}$. Ern, A. Suna, T. Tomkiewicz, P. Avakian, and R. P. Groff, Phys. Rev. B 5, 3222 (1972).

${ }^{9}$ M. D. Fayer and C. B. Harris, Phys. Rev. B 9, 748 (1974).

${ }^{10}$ R. Zwanzig, Ann. Rev. Phys. Chem. 16, 67 (1965).

${ }^{11}$ M. A. El-Sayed, J. Chem. Phys. 54, 680 (1971), and references therein.

${ }^{12}$ See, for example, J. Schmidt, D. A. Antheunis, and J. H. van der Waals, Mol, Phys. 22, 1 (1971).

${ }^{13}$ M. D. Fayer and C. B. Harris, Chem. Phys. Lett. 25, 149 (1974)。

${ }^{14} H_{\text {}}$ Brenner, J. C. Brock, M. D. Fayer, and C. B. Harris, Chem. Phys. Lett. 33, 471 (1975); M. T. Lewellyn, A. H. Zewail and C. B. Harris, J. Chem. Phys. 63, 3687 (1975).

${ }^{15}$ C. B. Harris, R. L。 Schlupp, and H. Schuch, Phys. Rev. Lett. 30, 1019 (1973).

${ }^{16}$ A. H. Francis and C. B. Harris, Chem. Phys. Lett. 9, 188 (1971).

${ }^{17}$ R. M. Hochstrasser and J.D. Whiteman, J. Chem, Phys. 56, 5945 (1972); J. D. Whiteman, Ph. D. thesis, Univ, of Pennsylvania, Philadelphia PA, 1971.

${ }^{18}$ R. M. Hochstrasser and A. H. Zewail, Chem. Phys. 4, 142 (1974).

${ }^{19}$ A. H. Francis and C. B. Harris, J. Chem. Phys. 57, 1050 (1972), and Ref. 16.

${ }^{20}$ A. M. Nishimura, A, H. Zewail, and C. B. Harris, J. Chem. Phys. 63, 1919 (1975).

${ }^{21}$ We should mention that other models ahve been put forth to solve the problem of Frenkel excitons on impure chains. Specifically, the impurity quenching parameters were added to the master equation of the exciton density matrix to describe the different limits of migration. This theoretical treatment by a Green function technique can be found in many references: R. M. Pearlstein, J. Chem. Phys. 56, 2431 (1972); R. P. Hemenger, K. L. Lindenberg, and R. M. Pearlstein, J. Chem. Phys. 60, 3271 (1974).

${ }^{22}$ R. E. Merrifield, J。 Chem。 Phys, 48, 4318 (1968).

${ }^{23} \mathrm{R}$. Schmidberger and $\mathrm{H}_{\text {。 }}$ C . Wolf, Chem. Phys. Lett. 16, 402 (1972).

${ }^{24}$ R. Schmidberger and H. C. Wolf, Chem. Phys. Lett. 25, 185 (1974).

${ }^{25} \mathrm{R}$. Schmidberger and H. C. Wolf, Chem. Phys, Lett. 32, 18,21 (1975).

${ }^{26}$ M. Sharnoff, Symp. Faraday Soc。 3, 137 (1969); see also Ref. 20 .

${ }^{27}$ A. H. Zewail and C. B. Harris, Chem. Phys. Lett. 28, 8 (1974).

${ }^{28}$ A. H. Zewail and C. B. Harris, Phys, Rev. B 11, 935 (1975).

${ }^{29}$ A. H. Zewail and C. B. Harris, Phys. Rev. B 11, 952 (1975).

${ }^{30}$ It would be very interesting to measure the coherence length as a function of temperature. However, the large number of parameters involved makes the extraction of the temperature dependence from our data difficult. In fact, in the relatively low temperature limit we have assumed that $K_{\varepsilon}, K_{t}$, and $K_{i}$ are temperature independent. The validity of this assumption was checked by the agreement between the experimental results and the model equations over the whole temperature range (see text). 